\title{
Inpatient Addiction Medicine Consultation and Post-Hospital Substance Use Disorder Treatment Engagement: a Propensity-Matched Analysis
}

\author{
Honora Englander, $M D^{7}{ }_{\mathbb{D}}$, Konrad Dobbertin, $M P H^{2}$, Bonnie K. Lind, $P h D^{2}$, \\ Christina Nicolaidis, $M D, M P H^{1,3}$, Peter Graven, $P h D^{7}$, Claire Dorfman, $B A^{4}$, and \\ $P$. Todd Korthuis, MD, MPH
}

\begin{abstract}
'Department of Medicine, Oregon Health \& Science University, Portland, OR, USA; ${ }^{2}$ Center for Health Systems Effectiveness, Oregon Health \& Science University, Portland, OR, USA; ${ }^{3}$ Portland State University School of Social Work, Portland, OR, USA; ${ }^{4}$ Oregon Clinical and Translational Research Institute, Oregon Health \& Science University, Portland, OR, USA.
\end{abstract}

BACKGROUND: Hospitalizations due to medical and surgical complications of substance use disorder (SUD) are rising. Most hospitals lack systems to treat SUD, and most people with SUD do not engage in treatment after discharge.

OBJECTIVE: Determine the effect of a hospital-based addiction medicine consult service, the Improving Addiction Care Team (IMPACT), on post-hospital SUD treatment engagement.

DESIGN: Cohort study using multivariable analysis of Oregon Medicaid claims comparing IMPACT patients with propensity-matched controls.

PARTICIPANTS: 18-64-year-old Oregon Medicaid beneficiaries with SUD, hospitalized at an Oregon hospital between July 1, 2015, and September 30, 2016. IMPACT patients $(n=208)$ were matched to controls $(n=$ 416) using a propensity score that accounted for SUD, gender, age, race, residence region, and diagnoses.

INTERVENTIONS: IMPACT included hospital-based consultation care from an interdisciplinary team of addiction medicine physicians, social workers, and peers with lived experience in recovery. IMPACT met patients during hospitalization; offered pharmacotherapy, behavioral treatments, and harm reduction services; and supported linkages to SUD treatment after discharge.

OUTCOMES: Healthcare Effectiveness Data and Information Set (HEDIS) measure of SUD treatment engagement, defined as two or more claims on two separate days for SUD care within 34 days of discharge.

RESULTS: Only $17.2 \%$ of all patients were engaged in SUD treatment before hospitalization. IMPACT patients engaged in SUD treatment following discharge more frequently than controls $(38.9 \%$ vs. $23.3 \%, p<0.01$; aOR 2.15 , 95\% confidence interval [CI] 1.29-3.58). IMPACT participation remained associated with SUD treatment engagement when limiting the sample to people who were not engaged in treatment prior to hospitalization (aOR 2.63; 95\% CI 1.46-4.72).

Electronic supplementary material The online version of this article (https://doi.org/10.1007/s11606-019-05251-9) contains supplementary material, which is available to authorized users.

Published online August 13, 2019
CONCLUSIONS: Hospital-based addiction medicine consultation can improve SUD treatment engagement, which is associated with reduced substance use, mortality, and other important clinical outcomes. National expansion of such models represents an opportunity to address an enduring gap in the SUD treatment continuum.

KEY WORDS: substance-related disorders; hospitalization; substance use treatment; Medicaid.

J Gen Intern Med 34(12):2796-803

DOI: $10.1007 / \mathrm{s} 11606-019-05251-9$

(c) Society of General Internal Medicine 2019

\section{INTRODUCTION}

Hospitals are increasingly filled with people suffering from medical complications of substance use disorders (SUD). ${ }^{1,2}$ One in seven patients in general hospitals has a substance use disorder, ${ }^{3}$ and hospitalizations for opioid-and methamphetamine-related diagnoses are surging. ${ }^{4-6}$ Patients with SUD have longer lengths of stay, higher costs, ${ }^{6}$ and higher readmission rates. ${ }^{3}$ Over the last decade, costs related to SUD-associated hospitalizations have quadrupled, with an estimated $\$ 15$ billion due to hospitalizations related to opioid use disorder in 2012 alone. $^{2}$

Despite frequent hospitalizations and high healthcare costs, most hospitalized patients with SUD are not engaged in SUD care before hospitalization, and most hospitals do not treat SUD during the acute inpatient encounter. ${ }^{2,7}$ Failure to treat SUD in hospitals leads to untreated withdrawal, failure to complete recommended medical therapy, and high rates of against medical advice discharge. ${ }^{8-10}$ Though there are decades of evidence showing the effectiveness of medication for opioid $^{11}$ and alcohol use disorders, ${ }^{12}$ most hospitals lack expertise and systems to treat SUD and do not effectively connect people to treatment after discharge. ${ }^{13}$ Barriers include lack of addiction medicine expertise, regulatory concerns, stigma, and silos between hospital and community SUD treatment settings. ${ }^{8,14}$ 
Hospitalization is a crucial potential juncture to engage outof-treatment adults in SUD care. ${ }^{15,16}$ Hospitalized patients with SUD have nearly twice the rate of hospital readmission and repeat ED use within 30 days, ${ }^{3}$ compared with those without SUD. And the immediate period after hospital discharge is particularly high risk for drug-related death and suicide. ${ }^{17}$ SUD treatment engagement is associated with reduced substance use severity, ${ }^{18-21}$ higher employment and wages, ${ }^{22}$ less future criminal involvement, ${ }^{21}$ reduced overdose rates, ${ }^{11}$ and reduced all-cause mortality. ${ }^{11,23}$ Hospitalization can be a reachable moment to initiate and coordinate SUD care. Although medical and surgical inpatients do not come to the hospital seeking addiction care, a study of hospitalized adults at our hospital found that over half of people with highrisk alcohol use and over two-thirds of people with high-risk drug use reported wanting to cut back or quit. ${ }^{24}$ Interventions to improve post-discharge SUD treatment engagement are likely to improve outcomes for people with SUD.

Despite a growing literature of the feasibility and importance of hospital-based addiction care, ${ }^{25-29}$ there are no studies using robust causal inference methods to evaluate the effects of inpatient addiction medicine consultation on SUD treatment engagement after discharge. A quasi-experimental study at a single Boston academic medical center comparing patients who received inpatient addiction medicine consultation with those who did not found that patients seen by the consult service were more likely to report SUD treatment engagement at 30 and 90 days after discharge. ${ }^{30}$ While promising, this study relied on self-reported outcomes and had $34 \%$ loss to follow-up at 30 days and $47 \%$ at 90 days. Additional rigorous evaluations to understand the effect of hospital-based addiction medicine care on post-hospital community SUD treatment are critical for broader adoption and dissemination.

We implemented a previously described interprofessional hospital-based addiction medicine consult service called the Improving Addiction Care Team (IMPACT). ${ }^{8,24,31,32}$ IMPACT engages adults with opioid, alcohol, methamphetamine, and other substances (excluding tobacco use disorder alone) during the reachable moment of hospitalization and provides rapid-access pathways to community SUD treatment and harm reduction support. The goal of this analysis was to assess posthospital SUD treatment engagement among Oregon Medicaid recipients who received IMPACT care compared with propensity score-matched controls. We hypothesized that IMPACT participation would be associated with greater post-hospital SUD treatment engagement.

\section{METHODS}

\section{Intervention}

IMPACT is a hospital-based addiction medicine consult service that includes care from addiction medicine physicians, social workers, and peers with lived experience in recovery. Inpatient medical and surgical providers and hospital social workers refer patients with known or suspected SUD to IMPACT. IMPACT is open to patients with any SUD (excluding people with tobacco use disorders alone) regardless of readiness to change or interest in treatment. Initially IMPACT served patients admitted to medicine, family medicine, cardiology services; or patients needing prolonged intravenous antibiotics admitted to any hospital service. IMPACT expanded to include surgical services in July 2017. IMPACT medical providers and social workers perform an initial comprehensive assessment, including DSM-5 SUD diagnosis assessment; elicit patient-centered goals around the acute hospitalization and SUD; initiate SUD treatment, including pharmacotherapy and behavioral treatments; and offer harm reduction services. IMPACT includes robust referral pathways to post-hospital SUD care and, in some cases, forges relationships with rural SUD providers to coordinate post-hospital care. ${ }^{24,31,32}$ Earlier work provides a detailed description of the interprofessional team and intervention. ${ }^{24,32}$

During the study window, IMPACT included 0.7 full-time equivalent (FTE) clinical physician time shared among 5 physicians, 1 social worker, and 2 peer recovery mentors. IMPACT physicians documented a median value of 2 patient visits (range 1-33) and social workers a median 3 (1-33) patient visits per hospitalization. Intervention intensity depended on patient needs, hospital length of stay, and IMPACT capacity.

\section{Setting and Study Design}

Earlier research found that IMPACT created a system-level change by reframing addiction as a treatable chronic disease, increasing provider understanding of how to engage and treat patients with SUD, and creating rapid pathways to care. ${ }^{8}$ As such, a patient- or provider-level randomized trial would not adequately isolate the intervention effect. Thus, we conducted a multivariable analysis comparing intervention patients with propensity-matched controls across Oregon hospitals. We used Oregon Medicaid claims data, obtained under a data use agreement with the Oregon Health Authority, that included physical/behavioral health and pharmacy claims in calendar years 2015-2016. This study was approved by the Oregon Health \& Science University (OHSU) Institutional Review Board.

\section{Population}

All participants were 18-64 years old with Oregon Medicaid insurance, had at least one SUD diagnosis (not including tobacco use disorder), and were hospitalized at an Oregon hospital between July 1, 2015, and September 30, 2016. IMPACT patients had confirmed or suspected SUD and were referred by medical and surgical providers at a large academic center in Portland, OR. Interest in SUD treatment was not required for IMPACT participation. Patients initially referred for IMPACT evaluation were excluded if a DSM-5 SUD diagnosis was not confirmed. We included individuals with 


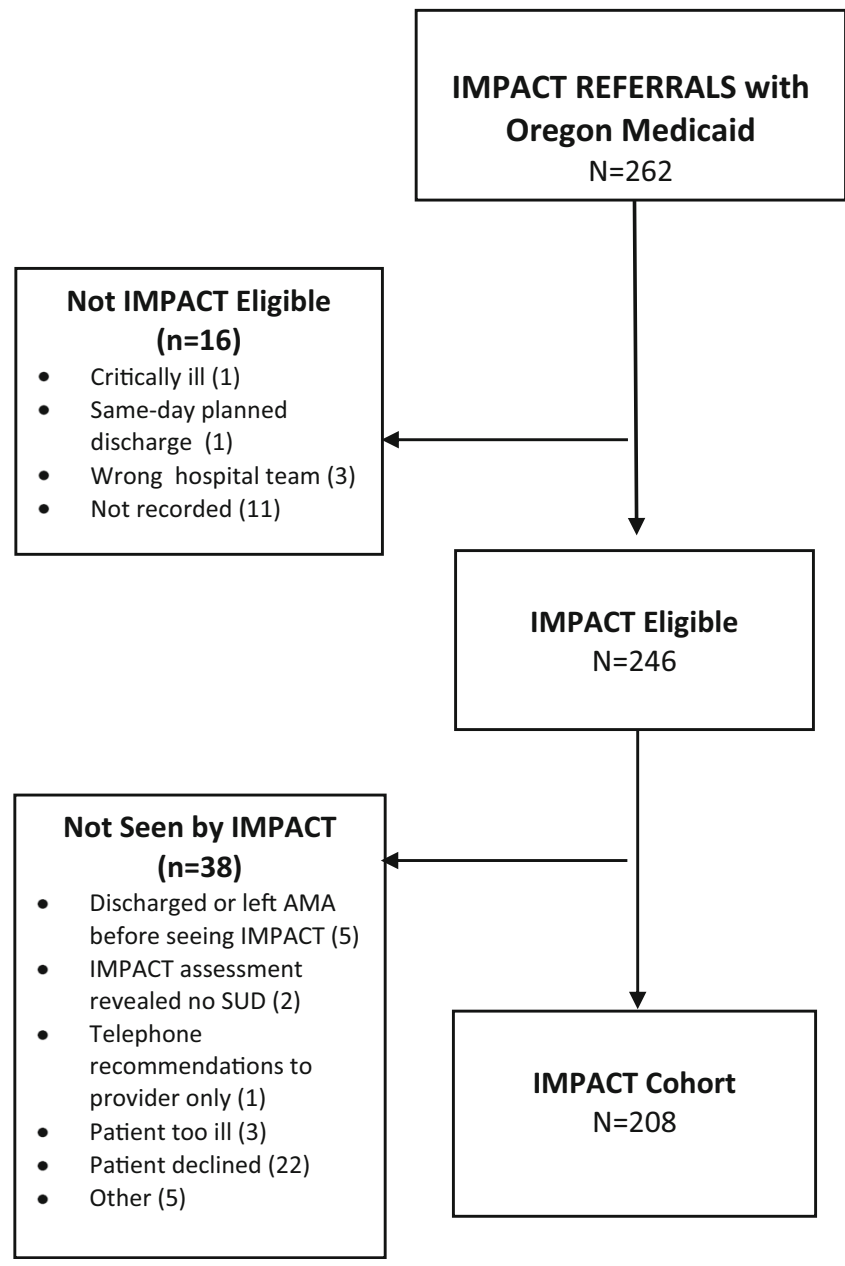

Figure 1 IMPACT participant flow diagram.

past SUD treatment because acute illness and hospitalization can interrupt SUD treatment ${ }^{33}$ and precipitate return to use, and IMPACT coordinates and supports care continuity for hospitalized patients already engaged in treatment. During the study window, IMPACT had 357 referrals. Of those, 269 (75.3\%) had Oregon Medicaid, of which 264 were eligible for IMPACT and 208 were seen by IMPACT and comprise the IMPACT cohort (Fig. 1).

We used propensity score matching to reduce bias by ensuring that cases and controls had comparable covariate distributions. ${ }^{34}$ First, we calculated a propensity score using all inpatient admissions with an SUD diagnosis. We calculated a propensity score using backward stepwise logistic regression and accounted for SUD type, gender, age, race, residence region, and diagnoses, including the Chronic Illness \& Disability Payment System (CDPS) ${ }^{35}$ risk score, which consists of 17 distinct disease categories that include physical and psychiatric diagnoses. Next, we matched IMPACT participants to potential controls matching on mutually exclusive SUD categories. For example, we matched patients with cooccurring opioid and methamphetamine use only to controls with co-occurring opioids and methamphetamine use. This allowed us to account for polysubstance use. Then, within each exactly matched SUD group, we nearest-neighbor matched each IMPACT patient to two controls, without replacement, based on propensity score.

\section{Measures}

The primary outcome, was the Healthcare Effectiveness Data and Information Set (HEDIS) National Committee for Quality Assurance (NCQA) definition of post-discharge SUD treatment engagement, ${ }^{36}$ which includes two or more of the following occurring on at least two separate days within 34 days of discharge: (1) a filled prescription for medication treatment (e.g., buprenorphine), (2) a procedure code for SUD treatment (e.g., methadone administration at an opioid treatment program, behavioral treatment for stimulant use disorder), or (3) a clinic visit with an SUD ICD-9 or ICD-10 code (Appendix in the Electronic Supplementary Material (ESM)). Filled prescriptions were limited to FDA-approved medications for SUD (excluding medication for tobacco use disorder). Medications dispensed from a hospital pharmacy at the time of discharge (for example, a bridging prescription for buprenorphine) do not appear in Oregon Medicaid claims; instead, they are included as part of the inpatient admission. In general, IMPACT's practice is to provide a 7-14-day bridge prescription for buprenorphine and up to 28 days for oral naltrexone or acamprosate at hospital discharge. A single prescription in the 34 days after discharge or one-time administration of a long-acting injectable medication would appear as a single encounter and not meet the criteria for HEDIS treatment engagement. Both of these factors might lead to an underestimate of post-hospital SUD treatment engagement using our primary outcome.

Covariates adjusted for in the final model included SUD treatment engagement in the 34 days prior to hospitalization, SUD type (opioid SUD vs. non-opioid SUD), gender, age, race/ethnicity, Portland tri-county area residence as a surrogate marker for urban versus non-urban healthcare systems, Medicare Severity Diagnosis-Related Group (MS-DRG) ${ }^{37}$ category, and Chronic Illness \& Disability Payment System risk score. $^{35}$

\section{Analysis}

We used multivariable logistic regression to evaluate the effect of IMPACT participation on treatment engagement. Due to small counts for many of the MS-DRGs, we collapsed them into clinically meaningful groups with sufficient numbers to model. High prevalence of polysubstance use led to sizeable overlap when we analyzed the effect of individual substances separately. Hence, we separated patients with any opioid use versus others because of the existence of more effective treatment options for opioid use disorder. Due to concerns that post-hospital treatment engagement could be driven by prior SUD treatment, we also conducted a sensitivity analysis modeling engagement among only those patients who had not engaged in SUD treatment in the 34 days prior to 
Table 1 Patient Characteristics: IMPACT Versus Controls

\begin{tabular}{|c|c|c|c|c|}
\hline & IMPACT, $N=208$ & Controls, $N=416$ & Total, $N=624$ & $p$ value $^{\mathrm{a}}$ \\
\hline Characteristic & $N(\%)$ & & & \\
\hline Prior SUD treatment ${ }^{\mathrm{b}}$ & $37(17.8)$ & $70(16.8)$ & $107(17.2)$ & 0.76 \\
\hline \multicolumn{5}{|l|}{ SUD type } \\
\hline Non-opioid SUD ${ }^{\mathrm{C}}$ & $146(70.2)$ & $292(70.2)$ & $438(70.2)$ & 1.00 \\
\hline Any opioid SUD ${ }^{d}$ & $62(29.8)$ & $124(29.8)$ & $186(29.8)$ & 1.00 \\
\hline Male & $127(61.1)$ & $249(59.9)$ & $376(60.3)$ & 0.77 \\
\hline Age & & & & 0.19 \\
\hline $18-39$ years & $102(49.0)$ & $181(43.5)$ & $283(45.4)$ & \\
\hline $40-64$ years & $106(51.0)$ & $235(56.5)$ & $341(54.7)$ & \\
\hline White non-Hispanic & $160(76.9)$ & $341(82.0)$ & $501(80.3)$ & 0.14 \\
\hline Portland tri-county area ${ }^{\mathrm{e}}$ & $172(82.7)$ & $295(70.9)$ & $467(74.8)$ & $<0.01$ \\
\hline \multicolumn{4}{|l|}{ MS-DRG category } & 0.01 \\
\hline Alcohol and related complications & $44(21.2)$ & $86(20.7)$ & $130(20.8)$ & \\
\hline Endocarditis, osteomyelitis, sepsis & $65(31.3)$ & $116(27.9)$ & $181(29.0)$ & \\
\hline Skin and soft tissue infections & $16(7.7)$ & $10(2.4)$ & $26(4.2)$ & \\
\hline Non-valvular cardiovascular & $13(6.3)$ & $17(4.1)$ & $30(4.8)$ & \\
\hline Orthopedic/joint & $9(4.3)$ & $20(4.8)$ & $29(4.7)$ & \\
\hline Other & $61(29.3)$ & $167(40.1)$ & $228(36.5)$ & \\
\hline Mean (SD) & & & & \\
\hline CDPS risk score ${ }^{\mathrm{g}}$ & $4.1(2.5)$ & $4.08(2.1)$ & $4.09(2.2)$ & 0.93 \\
\hline
\end{tabular}

IMPACT, Improving Addiction Care Team; SUD, substance use disorder

${ }^{a}$ We used $\chi^{2}$ for categorical variables and T-test for CDPS risk score

${ }^{b}$ Prior treatment defined as $\geq 2$ encounters for SUD treatment on 2 separate days in 34 days before admission. Codes used to define SUD treatment encounters are detailed in the Appendix in the ESM and include (1) a filled prescription for medication treatment, (2) a procedure code for SUD treatment, or (3) a clinic visit with an SUD ICD-9 or ICD-10 code

${ }^{c}$ Includes alcohol alone, stimulant alone, alcohol + stimulant, other

${ }^{d}$ Includes opioids alone, opioids + alcohol, opioids + stimulant, opioids + alcohol + stimulant

${ }^{e}$ Clackamas, Multnomah, and Washington counties

${ }^{f}$ Medicare Severity Diagnosis Related Group (MS-DRG)

${ }^{g}$ Chronic Illness \& Disability Payment System (CDPS), continuous variable

hospitalization. We performed data management and statistical analysis using Stata version 15.1.

\section{RESULTS}

Cases $(n=208)$ and controls $(n=416)$ had similar gender, race and ethnicity, rural residence, age, and mean CDPS risk score distribution (Table 1). $60.3 \%$ were male, $54.7 \%$ were 40 years or older, and $29.8 \%$ had any opioid SUD diagnosis. More cases resided in the Portland tri-county area $(82.7 \%$ vs. $70.9 \%$ ). More controls had DRGs in the heterogeneous "other" category $(40.1 \%$ vs. $29.3 \%)$. Only $17.2 \%$ of all patients were engaged in treatment in the 34 days prior to hospitalization, with similar rates among IMPACT (12.5-19.2\%) and controls $(12.3-16.0 \%)$. Of the 208 IMPACT patients, 31 received a discharge prescription for buprenorphine, 14 for oral naltrexone, 8 for acamprosate, and 18 received injectable naltrexone during hospitalization with a plan to continue. At the time of discharge, 56 had a plan to continue methadone maintenance. At 34 days, 1 person in IMPACT and 14 people in the control group had died.

In the primary analysis, IMPACT patients engaged in SUD treatment following discharge more frequently than controls (38.9\% vs. $23.3 \%, p<0.01$; adjusted odds ratio [aOR] 2.15 , 95\% confidence interval [CI] 1.29-3.58; Table 2). Predictably, those engaged in treatment before admission had substantially greater odds of receiving treatment after discharge (aOR 11.95; 95\% CI 6.31-22.65). A sensitivity analysis limiting the sample to those without pre-hospital SUD treatment showed similar results, with a significant association between IMPACT and engagement in SUD treatment (aOR 2.63, 95\% CI 1.46-4.72). Patients with opioid use disorder were more likely than those without an opioid use disorder to engage in treatment (aOR 1.83, 95\% CI 1.03-3.24).

The figure shows the unadjusted proportion of patients receiving treatment in the 6 months before hospitalization and within the 34 days after discharge. Treatment engagement is similar in the months before admission and increases following discharge, more for IMPACT patients than for controls (39.9\% vs. $23.3 \%, p<0.01)$ (Fig. 2 ).

\section{DISCUSSION}

Hospitalized Medicaid recipients with SUD who received inpatient addiction medicine consultation had greater odds of post-hospital SUD treatment engagement than matched controls. Few patients were engaged in SUD care prior to hospitalization, highlighting the role of hospitalization as a reachable moment. To our knowledge, this is most rigorously controlled study demonstrating the effectiveness of an inpatient addiction medicine consult service in improving SUD treatment engagement, one of the enduring gaps in the SUD treatment continuum. ${ }^{38}$

Our study extends previous research in several important ways. First, propensity score-matched controls provide a rigorous comparison group which alleviates potential bias due to 
Table 2 Model Results: Patient Characteristics Associated with Treatment Engagement

\begin{tabular}{|c|c|c|c|c|c|c|c|c|c|}
\hline & & \multicolumn{5}{|c|}{ Unadjusted analyses } & \multicolumn{3}{|c|}{ Adjusted analyses } \\
\hline & & \multirow{2}{*}{$\begin{array}{l}\text { Total } \\
N\end{array}$} & \multicolumn{2}{|c|}{$\begin{array}{l}\text { Engaged in } \\
\text { treatment }^{\mathrm{a}}\end{array}$} & \multicolumn{2}{|c|}{$\begin{array}{l}\text { Not engaged in } \\
\text { treatment }\end{array}$} & \multirow[t]{2}{*}{$\mathbf{a O R}^{\mathbf{b}}$} & \multicolumn{2}{|c|}{$95 \% \mathrm{CI}$} \\
\hline \multicolumn{2}{|l|}{ Characteristic } & & $N$ & $(\%)$ & $\boldsymbol{N}$ & $(\%)$ & & & \\
\hline \multicolumn{2}{|l|}{ Control } & 416 & 97 & $(23.3)$ & 349 & $(76.7)$ & Ref. & - & - \\
\hline \multicolumn{2}{|c|}{ IMPACT } & 208 & 81 & (38.9) & 127 & (61.1) & 2.15 & 1.29 & 3.58 \\
\hline \multicolumn{2}{|c|}{ No prior SUD treatment ${ }^{\mathrm{c}}$} & 517 & 101 & $(19.5)$ & 416 & $(80.5)$ & Ref. & - & - \\
\hline \multicolumn{2}{|c|}{ Prior SUD treatment } & 107 & 77 & $(72.0)$ & 30 & $(28.0)$ & 12.12 & 6.44 & 22.82 \\
\hline \multirow[t]{2}{*}{ SUD type } & Non-opioid SUD ${ }^{\mathrm{d}}$ & 186 & 36 & (19.4) & 150 & $(80.6)$ & Ref. & - & - \\
\hline & Any opioid SUD & 438 & 142 & $(32.4)$ & 296 & $(67.58)$ & 1.83 & 1.03 & 3.24 \\
\hline \multirow[t]{2}{*}{ Gender } & Female & 248 & 73 & $(29.4)$ & 175 & $(70.6)$ & Ref. & - & - \\
\hline & Male & 376 & 105 & (27.9) & 271 & $(72.1)$ & 1.05 & 0.63 & 1.74 \\
\hline \multirow[t]{2}{*}{ Age (years) } & $18-39$ & 283 & 83 & $(29.3)$ & 200 & $(70.7)$ & Ref. & - & - \\
\hline & $40-64$ & 341 & 95 & $(27.9)$ & 246 & $(72.1)$ & 0.83 & 0.48 & 1.40 \\
\hline \multirow[t]{2}{*}{ Race and ethnicity } & Other & 123 & 38 & $(30.9)$ & 85 & (69.1) & Ref. & - & - \\
\hline & White non-Hispanic & 501 & 140 & $(27.9)$ & 361 & $(72.1)$ & 0.92 & 0.53 & 1.62 \\
\hline \multirow[t]{2}{*}{ Region } & Non-tri-county area & 157 & 27 & $(17.2)$ & 130 & $(82.8)$ & Ref. & - & - \\
\hline & Portland tri-county area ${ }^{\mathrm{f}}$ & 467 & 151 & $(32.3)$ & 316 & $(67.7)$ & 1.74 & 0.90 & 3.34 \\
\hline \multirow[t]{6}{*}{ MS-DRG category ${ }^{\mathrm{g}}$} & Alcohol and related complications & 130 & 35 & $(26.9)$ & 95 & $(73.1)$ & Ref. & - & - \\
\hline & Endocarditis, osteomyelitis, sepsis & 181 & 54 & $(29.8)$ & 127 & $(70.2)$ & 1.19 & 0.62 & 2.28 \\
\hline & Skin and soft tissue infections & 26 & 12 & $(46.2)$ & 14 & $(53.8)$ & 2.36 & 0.88 & 6.32 \\
\hline & Non-valvular cardiovascular & 30 & 13 & $(43.3)$ & 17 & $(56.7)$ & 2.07 & 0.80 & 5.36 \\
\hline & Orthopedic/joint & 29 & 6 & $(20.7)$ & 23 & (79.3) & 0.49 & 0.12 & 2.02 \\
\hline & Other & 228 & 58 & $(25.4)$ & 170 & $(74.6)$ & 0.98 & 0.49 & 1.94 \\
\hline \multicolumn{10}{|l|}{ Mean (SD) } \\
\hline \multicolumn{2}{|l|}{ CDPS risk score ${ }^{\mathrm{h}}$} & & 4.04 & $(2.1)$ & 4.11 & $(2.3)$ & 1.05 & 0.93 & 1.19 \\
\hline
\end{tabular}

IMPACT, Improving Addiction Care Team; SUD, substance use disorder

${ }^{a}$ Treatment defined as $\geq 2$ encounters for SUD treatment on 2 separate days within 34 days of discharge; Healthcare Effectiveness Data and Information Set (HEDIS) measure

${ }^{b}$ Adjusted odds ratio (OR), adjusted for all variables in Table 2

${ }^{c}$ Prior treatment defined as $\geq 2$ encounters for SUD treatment on 2 separate days in 34 days before admission

${ }^{d}$ Includes alcohol alone, stimulant alone, alcohol + stimulant, other

${ }^{e}$ Includes opioids alone, opioids + alcohol, opioids + stimulant, opioids + alcohol + stimulant

${ }^{f}$ Clackamas, Multnomah, and Washington counties

${ }^{g}$ Medicare Severity Diagnosis-Related Group (MS-DRG)

${ }^{h}$ Chronic Illness \& Disability Payment System (CDPS), continuous variable

systematic differences in treatment groups, ${ }^{39}$ and we also controlled for known confounders. Our work builds on a quasi-experimental study at a Boston academic medical center that compared patients who received inpatient addiction medicine consultation with those who did not, finding that patients seen by the inpatient consult service were more likely to report SUD treatment engagement at 30 and 90 days after

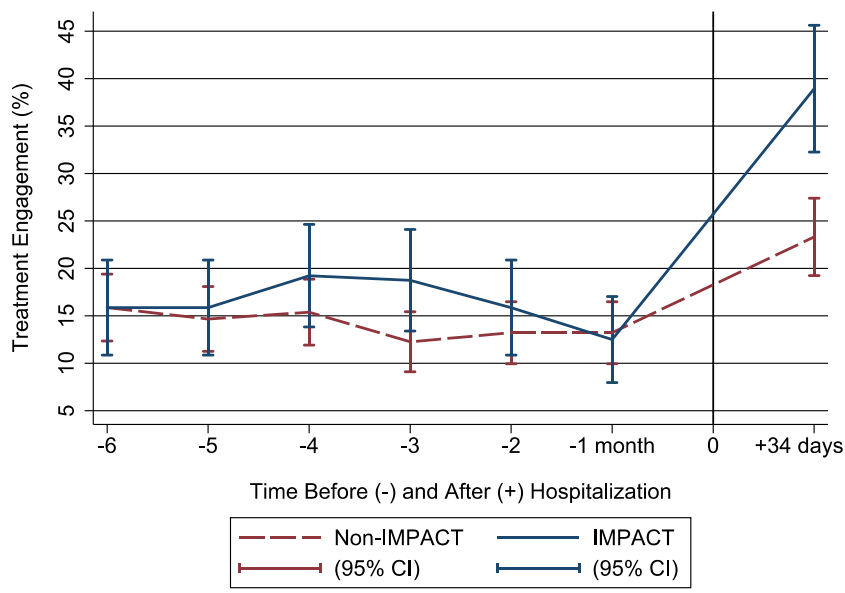

Figure 2 Substance use disorder treatment engagement over time, comparing IMPACT and controls. discharge. ${ }^{30}$ While promising, this study was quasi-experimental, relied on self-reported outcomes, and had high loss to follow-up (34\% at 30 days, $47 \%$ at 90 days). Second, whereas earlier studies ${ }^{25-27}$ measure engagement as a single SUD-related encounter, ours ties hospital-initiated care to multiple post-hospital treatment encounters using the NCQA HEDIS measure. Third, our study measured treatment engagement state-wide across any setting, whereas prior studies measured engagement by self-report or referral to a single SUD treatment location. ${ }^{25,27}$ Finally, claims data provide a more reliable measure of treatment engagement than selfreport.

Patients with opioid use disorder (OUD) had greater odds of engagement than those with non-opioid SUD. We hypothesize that this may be due to greater uptake and effectiveness of medications for OUD compared with non-opioid SUD, particularly for methamphetamine use disorder.

Treatment prior to admission was, unsurprisingly, associated with substantial increased odds of treatment engagement following hospitalization. One might hypothesize that the effect of an intervention such as IMPACT is driven by prior treatment engagement; however, a sensitivity analysis showed IMPACT had a durable effect among patients without prior treatment engagement. This highlights a potential role of inpatient addiction medicine consult services in facility 
treatment retention by avoiding treatment interruption during hospitalization and return to use - another major gap in the SUD treatment continuum.

Our study has several important implications. First, hospitals can and should address SUD. Current hospital practice, which treats acute medical problems resulting from SUD without addressing the underlying cause, is analogous to performing an amputation for a diabetic foot infection without providing insulin or referring to outpatient diabetes care. The finding that inpatient addiction medicine care increases posthospital treatment engagement further underscores the need to address SUD during hospitalization.

In our study, only $17 \%$ of people were engaged in treatment prior to hospitalization. This is consistent with national trends showing that only $7.7 \%$ of people with any past-year SUD received treatment. ${ }^{40}$ Current reform efforts have focused largely on integrating addiction treatment in primary care. These are critical reforms, and long-term success of hospital-based interventions depends on availability of community treatment. However, integrating SUD care in hospitals should not be overlooked. Hospitals have potential to engage people who are not engaged in primary care, who are medically complex, whose SUD may be particularly severe, and who may be high healthcare utilizers.

Doubling the odds of treatment engagement, with $39 \%$ of IMPACT participants engaging in post-hospital SUD care, is important. Our outcome measure may underestimate treatment engagement. However, the patients that did not connect to treatment warrant consideration. Not all hospitalized adults with SUD want treatment, highlighting our continued duty to implement harm reduction practices in hospitals and across communities. Ample evidence supports that harm reduction practices make substance use safer and ultimately can engage people in treatment. ${ }^{41-44}$ However, some patients may have wanted to engage in treatment and been unsuccessful because of limited transportation, housing, criminal justice involvement, and medical illness. Hospital interventions can be catalysts for community-level change, but alone they cannot address structural barriers such as federal regulations limiting methadone access, banning of medication for opioid use disorder in jails, and the need for more buprenorphine providers. Rural treatment access is another important consideration. Though it did not meet the statistical threshold for significance, patients with SUD who resided in areas outside of metropolitan Portland less commonly engaged in SUD treatment following discharge, consistent with lack of treatment providers, transportation, and other barriers that limit access to SUD care in rural America. ${ }^{45,46}$ Finally, medical frailty presents a significant challenge to SUD treatment engagement. Barriers occur at the level of SUD treatment, where the physical demands of standing in line for methadone or participating in groups can represent insurmountable barriers. And postacute care settings such as skilled nursing facilities (SNFs) often force people to choose physical rehabilitation or SUD treatment (also often denying people access to SNF altogether based on a history of SUD) ${ }^{26}$ In this regard, hospital-based addiction medicine consult services have potential to improve SUD treatment retention and relapse prevention through care planning-another major gap in the SUD treatment continuum.

Our study has several important limitations. First, claims data are collected primarily for billing and may not accurately reflect all diagnoses. Further, claims do not include nonbillable SUD services such as 12-step meetings, syringe exchange services, or prescriptions dispensed on the day of discharge from a hospital pharmacy - a common practice for IMPACT that potentially underestimates its effect. Second, the study is not a randomized controlled trial and thus may be influenced by selection bias or unknown confounders. Due to complexities related to when IMPACT was available across different services, we do not have data on the total number of patients that could have been eligible for IMPACT. It is possible that providers referred patients who would be more or less likely than controls to engage in SUD treatment. For example, providers may have preferentially referred patients who expressed an interest in treatment. Alternatively, providers may have referred patients who declined usual care social work SUD referrals or who had severe medical or behavioral complications (e.g., disruptive behaviors including active in-hospital drug use, leaving against medical advice) associated with lower likelihood of treatment engagement. We mitigated this by (1) making IMPACT broadly inclusive (excluding only those who refused to speak with us and reducing the risk that only motivated patients entered the study), (2) propensity score matching, which can approximate randomization and adjust for unmeasured confounders, ${ }^{47,48}$ (3) adjusting for covariates and pre-hospital SUD treatment, and (4) using a stringent outcome which may underestimate the true effect of IMPACT. Third, we limited our study population to adults with Oregon Medicaid; however, Medicaid patients comprise most hospitalized patients with SUD. ${ }^{49}$ Fourth, this was not a blinded study; however, we do not expect blinding to affect assessment of the outcome, which was measured using administrative data. Fifth, Medicaid claims data does not reliably include important social factors such as housing and transportation, so we were unable to control for these. Sixth, we were unable to assess follow-up among people who lost Medicaid insurance in the 34 days after discharge, which likely had a minimal effect, and in those who moved out of Oregon following discharge. Because IMPACT occasionally supported people to connect with supportive family and/or treatment out-of-state, this may have underestimated SUD engagement in the IMPACT group. Finally, our study was performed in a single state with low racial and ethnic diversity and was among the first states to expand Medicaid, which may limit generalizability.

Our study shows that hospitalization can be a place to initiate and engage people in SUD care; however, more research is needed to understand how to best support long-term 
treatment retention. Recently hospitalized adults may have different treatment needs. Future research examining treatment retention among recently discharged adults with SUD is warranted. This research should include those in treatment before admission and those who initiated treatment during hospitalization.

Future research should define which components of hospital-based addiction care such as IMPACT are most important: for example, for which patients with opioid use disorder is a physician prescribing medication or linking to community treatment sufficient, and which patients need peer support or social work intervention? Additional research is also needed to understand how to adapt an IMPACT model to different settings with different resources. Finally, future research is needed to assess the effect of hospital-based addiction medicine consultation on patient experience, SUD severity, against medical advice discharge rates, physical health outcomes, hospital readmissions, healthcare costs, and mortality.

\section{CONCLUSIONS}

Hospital-based addiction medicine care can improve SUD treatment engagement. National expansion of such models represents an opportunity to address one of the enduring gaps in the SUD treatment continuum.

Acknowledgments: Authors would like to acknowledge the entire IMPACT clinical and research teams. Specifically, they would like to thanks Alisa Patten and Devin Collins for their contributions to this paper, and Dr Melissa Weimer, Daren Ford, and O'Nesha Cochran Dumas for their dedication as the original IMPACT clinical team

Corresponding Author: Honora Englander, MD; Department of MedicineOregon Health \& Science University, Portland, OR, USA (e-mail: englandh@ohsu.edu).

Funding Information This study was funded by the OHSU School of Medicine. IMPACT is funded by Oregon Health \& Science University and CareOregon. The OHSU School of Medicine funded IMPACT evaluation activities. Dr. Korthuis' time was supported by the National Institutes of Health and National Institute on Drug Abuse (UG3DA044831, UG1DA015815). This publication was made possible with support from the Oregon Clinical and Translational Research Institute (OCTRI), grant number UL1TR002369 from the National Center for Research Resources (NCRR), a component of the National Institutes of Health (NIH), and NIH Roadmap for Medical Research.

\section{Compliance with Ethical Standards:}

This study was approved by the Oregon Health \& Science University (OHSU) Institutional Review Board.

Conflict of Interest: The authors declare that they do not have a conflict of interest.

Disclaimer: The funders had no role in the design, conduct, and reporting of this study.

\section{REFERENCES}

1. Tedesco D, Asch SM, Curtin C, Hah J, McDonald KM, Fantini MP, et al. Opioid abuse and poisoning: trends in inpatient and emergency department discharges. Health Aff (Millwood) 2017;36(10):1748-53.

2. Ronan MV, Herzig SJ. Hospitalizations related to opioid abuse/ dependence and associated serious infections increased sharply, 200212. Health Aff (Millwood) 2016;35(5):832-7.

3. Walley AY, Paasche-Orlow M, Lee EC, Forsythe S, Chetty VK, Mitchell $\mathbf{S}$, et al. Acute care hospital utilization among medical inpatients discharged with a substance use disorder diagnosis. J Addict Med 2012;6(1):50-6.

4. Weiss A, Elixhauser A, Barrett M, Steiner C, Bailey M, O'Malley L. Opioid-related inpatient stays and emergency department visits by state, 2009-2014. Statistical Brief \#219. Healthcare Cost and Utilization Project. 2016. https://www.hcup-us.ahrq.gov/reports/statbriefs/ sb219-Opioid-Hospital-Stays-ED-Visits-by-State.jsp. Accessed May 21, 2019.

5. Winkelman TA, Admon LK, Jennings L, Shippee ND, Richardson CR, Bart G. Evaluation of amphetamine-related hospitalizations and associated clinical outcomes and costs in the United States. JAMA Netw Open 2018;1(6):e183758.

6. Schranz AJ, Fleischauer A, Chu VH, Wu LT, Rosen DL. Trends in drug use-associated infective endocarditis and heart valve surgery, 2007 to 2017: a study of statewide discharge data. Ann Intern Med. 2018 Dec 4. https://doi.org/10.7326/M18-2124

7. Naeger S, Ali MM, Mutter R, Mark TL, Hughey L. Prescriptions filled following an opioid-related hospitalization. Psychiatr Serv 2016;67(11): 1262-4.

8. Englander H, Collins D, Perry SP, Rabinowitz M, Phoutrides E, Nicolaidis C. "We've learned it's a medical illness, not a moral choice": qualitative study of the effects of a multicomponent addiction intervention on hospital providers' attitudes and experiences. J Hosp Med 2018;13(11):752-8.

9. Ti L , Ti L. Leaving the hospital against medical advice among people who use illicit drugs: a systematic review. Am J Public Health 2015;105(12):e53-9.

10. McNeil R, Small W, Wood E, Kerr T. Hospitals as a 'risk environment': an ethno-epidemiological study of voluntary and involuntary discharge from hospital against medical advice among people who inject drugs. Soc Sci Med 2014;105:59-66.

11. Sordo L, Barrio G, Bravo MJ, Indave BI, Degenhardt $\mathbf{L}$, Wiessing $\mathbf{L}$, et al. Mortality risk during and after opioid substitution treatment: systematic review and meta-analysis of cohort studies. BMJ (Clinical research ed). 2017;357:j1550.

12. Jonas DE, Amick HR, Feltner C, Bobashev G, Thomas $\mathbf{K}$, Wines $\mathbf{R}$, et al. Pharmacotherapy for adults with alcohol use disorders in outpatient settings: a systematic review and meta-analysis. JAMA. 2014;311(18): 1889-900.

13. Rosenthal ES, Karchmer AW, Theisen-Toupal J, Castillo RA, Rowley CF. Suboptimal addiction interventions for patients hospitalized with injection drug use-associated infective endocarditis. Am J Med 2016;129(5):481-5.

14. Winetsky D, Weinrieb RM, Perrone J. Expanding treatment opportunities for hospitalized patients with opioid use disorders. J Hosp Med 2018;13(1):62-4.

15. Saitz R. Treatment for opioid addiction must be offered in general hospitals: but how? J Addict Med 2019;13(2):83-4.

16. D'Onofrio G, O'Connor PG, Pantalon MV, Chawarski MC, Busch SH, Owens PH, et al. Emergency department-initiated buprenorphine/ naloxone treatment for opioid dependence: a randomized clinical trial. Jama. 2015;313(16):1636-44.

17. Merrall EL, Bird SM, Hutchinson SJ. A record-linkage study of drugrelated death and suicide after hospital discharge among drug-treatment clients in Scotland, 1996-2006. Addiction 2013;108(2):377-84.

18. Mattick RP, Breen C, Kimber J, Davoli M. Buprenorphine maintenance versus placebo or methadone maintenance for opioid dependence. Cochrane Database Syst Rev 2014;(2):CD002207.

19. Nielsen S, Larance B, Degenhardt L, Gowing L, Kehler C, Lintzeris N. Opioid agonist treatment for pharmaceutical opioid dependent people. Cochrane Database Syst Rev 2016;(5):Cd011117.

20. Saxon AJ, Ling W, Hillhouse M, Thomas C, Hasson A, Ang A, et al. Buprenorphine/naloxone and methadone effects on laboratory indices of liver health: a randomized trial. Drug Alcohol Depend 2013;128(12):71-6. 
21. Harris AH, Humphreys $\mathbf{K}$, Bowe T, Tiet $\mathbf{Q}$, Finney JW. Does meeting the HEDIS substance abuse treatment engagement criterion predict patient outcomes? J Behav Health Serv Res 2010;37(1):25-39.

22. Arria AM. Drug treatment completion and post-discharge employment in the TOPPS-II Interstate Cooperative Study. J Subst Abus Treat 2003;25(1):9-18.

23. Larochelle MR, Liebschutz JM, Zhang F, Ross-Degnan D, Wharam JF Opioid prescribing after nonfatal overdose and association with repeated overdose: a cohort study. Ann Intern Med 2016;164(1): 1-9.

24. Englander $\mathbf{H}$, Weimer $\mathbf{M}$, Solotaroff $\mathbf{R}$, Nicolaidis $\mathbf{C}$, Chan B, Velez $\mathbf{C}$, et al. Planning and designing the Improving Addiction Care Team (IMPACT) for hospitalized adults with substance use disorder. J Hosp Med 2017;12(5):339-42.

25. Liebschutz JM, Crooks D, Herman D, Anderson B, Tsui J, Meshesha $\mathbf{L Z}$, et al. Buprenorphine treatment for hospitalized, opioid-dependent patients: a randomized clinical trial. JAMA Intern Med 2014; 174(8): 1369-76.

26. Wakeman SE, Rich JD. Barriers to post-acute care for patients on opioid agonist therapy; an example of systematic stigmatization of addiction. J Gen Intern Med 2017;32(1):17-9.

27. Shanahan CW, Beers D, Alford DP, Brigandi E, Samet JH. A transitional opioid program to engage hospitalized drug users. J Gen Intern Med 2010;25(8):803-8.

28. Trowbridge $\mathbf{P}$, Weinstein ZM, Kerensky T, Roy P, Regan D, Samet JH, et al. Addiction consultation services - linking hospitalized patients to outpatient addiction treatment. J Subst Abus Treat 2017;79:1-5.

29. Fanucchi L, Lofwall MR. Putting Parity into Practice - Integrating Opioid-Use Disorder Treatment into the Hospital Setting. N Engl J Med 2016;375(9):811-3

30. Wakeman SE, Metlay JP, Chang Y, Herman GE, Rigotti NA. Inpatient addiction consultation for hospitalized patients increases post-discharge abstinence and reduces addiction severity. J Gen Intern Med 2017;32(8):909-16.

31. Englander H, Gregg J, Gullickson J, Cochran O, Colasurdo C, Alla J, et al. Recommendations for intergrating peer mentors in hospital-based addiction care. Subst Abus In press. https://doi.org/10.1080/ 08897077.2019.1635968

32. Englander H, Mahoney S, Brandt K, Brown J, Dorfman C, Nydahl A et al. Tools to support hospital-based addiction care: core components, values, and activities of the Improving Addiction Care Team. J Addict Med 2019;13(2):85-9.

33. Priest KC. Hospital-based services for opioid use disorder: a study of supply-side attributes. Dissertation and Theses. 2019. Paper 4829 Available from: https://pdxscholar.library.pdx.edu/open_access_etds/ 4829 .

34. Rubin DB. Using multivariate matched sampling and regression adjustment to control bias in observational studies. J Am Stat Assoc 1979;74(366):318-28.

35. Kronick R, Gilmer T, Dreyfus T, Lee L. Improving health-based payment for Medicaid beneficiaries: CDPS. Health Care Financ Rev 2000;21(3):29-64.

36. National Committee for Quality Assurance. Initiation and Engagement of Alcohol and Other Drug Abuse or Dependence Treatment (IET). 2017. [Cited 2019 June 7]. Available from: https://www.ncqa.org/hedis/measures/initiation-and-engagement-of-alcohol-and-other-drug-abuse-ordependence-treatment/.
37. Centers for Medicare \& Medicaid Services. ICD-10-CM/PCS MS-DRG v34.0 Definitions Manual. [cited 2019 Apr 12]. Available from: https:// www.cms.gov/ICD10Manual/version34-fullcode-cms/fullcode_cms/ P0001.html.

38. Socias ME, Wood E, Kerr T, Nolan S, Hayashi K, Nosova E, et al. Trends in engagement in the cascade of care for opioid use disorder, Vancouver, Canada, 2006-2016. Drug Alcohol Depend 2018;189:90-5.

39. Dehejia R, Wahba S. Propensity score matching methods for nonexperimental causal studies. Rev Econ Stat 2002;84:151-61.

40. Substance Abuse and Mental Health Services Administration. Key Substance Use and Mental Health Indicators in the United States: Results from the 2017 National Survey on Drug Use and Health (HHS Publication No. SMA 18-5068, NSDUH Series H-53). Rockville, MD 2018. [cited 2019 Apr 12]. Available from: https://www.samhsa.gov/data/ sites/default/files/cbhsq-reports/NSDUHFFR2017/NSDUHFFR2017. htm.

41. Potier C, Laprevote V, Dubois-Arber F, Cottencin O, Rolland B. Supervised injection services: what has been demonstrated? A systematic literature review. Drug Alcohol Depend 2014;145:48-68.

42. Abdul-guader AS, Feelemyer J, Modi S, Stein ES, Briceno A, Semaan $\mathbf{S}$, et al. Effectiveness of structural-level needle/syringe programs to reduce $\mathrm{HCV}$ and $\mathrm{HIV}$ infection among people who inject drugs: a systematic review. AIDS Behav 2013;17(9):2878-92.

43. Chou R, Korthuis PT, McCarty D, Coffin PO, Griffin JC, Davis-O'Reilly $\mathbf{C}$, et al. Management of suspected opioid overdose with naloxone in outof-hospital settings: a systematic review. Ann Intern Med 2017;167(12):867-75.

44. Thakarar $\mathbf{K}$, Weinstein $\mathbf{Z M}$, Walley AY. Optimising health and safety of people who inject drugs during transition from acute to outpatient care: narrative review with clinical checklist. Postgrad Med J 2016;92(1088):356-63.

45. Borders TF, Booth BM. Research on rural residence and access to drug abuse services: where are we and where do we go? J Rural Health 2007;23 Suppl:79-83

46. Ziller EC, Anderson NJ, Coburn AF. Access to rural mental health services: service use and out-of-pocket costs. J Rural Health 2010;26(3):214-24.

47. Deaton A, Cartwright N. Understanding and misunderstanding randomized controlled trials. Soc Sci Med 2018;210:2-21.

48. Frieden TR. Evidence for Health Decision Making - Beyond Randomized, Controlled Trials N Engl J Med 2017;377(5):465-75.

49. Weiss AJ, Heslin KC. Payers of opioid-related inpatient stays and emergency department visits nationally and by state, 2010 and 2015 . HCUP Statistical Brief \#239. Agency for Healthcare Research and Quality, Rockville (MD). 2018. [cited 2019 Apr 12]. Available from: https://www. hcup-us.ahrq.gov/reports/statbriefs/sb239-Opioid-Payer-HospitalStays-ED-Visits-by-State.jsp.

Publisher's Note Springer Nature remains neutral with regard to jurisdictional claims in published maps and institutional affiliations. 